\title{
Words To Live By: Investigating Navajo Naming Practices
}

\author{
Theodore Holland, Jr.
}

Duncansville, Pennsylvania

\begin{abstract}
The onomastic researcher who utilizes tape-recorded interviews as a source of information faces the immediate data management problems of how to process, organize, and reduce the rapidly proliferating accumulation of unorganized raw data afforded by interview transcripts and fieldnotes. THE ETHNOGRAPH ${ }^{\circledR}$, a program for the computer-assisted analysis of text-based data, was a valuable aid in an exploratory study of Navajo naming practices.
\end{abstract}

My fascination with names was about to lead me, on disturbingly short notice, to the most unanticipated of places. In April of 1991, as a new doctoral student, and wishing to speedily retire an internship requirement, I jumped at the chance to join Northwestern University's Ethnographic Field School. Thus it was that I would find myself thrust into a culture with which I had not the slightest familiarity. In mid-June I left Pennsylvania for the Navajo Reservation in Arizona. My task was to study Navajo names.

Four days of driving brought me to my destination, and every rest stop found me perusing my notes. Dozens of volumes at university libraries had yielded but a few pages here, a few paragraphs there. One could learn, to be sure, that a Navajo's names were subject to change, or that they were often based on an individual's behavioral or physical characteristics, residence location, or clan membership. Yet beyond such general statements, the majority of introductory, general, or biographical texts on the Navajo had little to offer in the way of substantive information relevant to naming practices.

Names 43.4 (December 1995):275-293

ISSN:0027-7738

(1) 1995 by The American Name Society 


\section{Names 43.4 (December 1995)}

Scholarly texts focusing on other topics (such as Navajo religion) offered additional fare, though again, naming practices entered as oblique references and only to the extent that they impinged upon such texts' primary foci. Young and Morgan (1987), for example, afforded several pages exampling "war" names and descriptive sobriquets; their goal of providing a dictionary and grammar of the Navajo language precluded an investigation of naming practices per se. Reichard (1928; 1950) provided a brief overview of Navajo names, as did The Navajo Yearbook (Bureau of Indian Affairs 1961). As well, texts specifically addressing ritual and cosmology proved obligatory, given that it is not possible to approach the deeper significance of Navajo names without penetrating somewhat into the spiritual milieu of their usage. Taken as a whole, however, the literature did not afford a sustained interrogation of Navajo personal names; generally absent was any clear depiction of the domain which they encompassed or of their shifting patterns of utilization or significance through time. Nonetheless, these texts allowed me to cobble together a tentative typology of Navajo names. With tape recorder in hand, that exploratory and ill-defined process called "fieldwork" began.

The researcher who uses tape-recorded conversation faces immediate information management problems. In my instance, a single interview usually yielded some three dozen pages of transcripts; multiply that by several dozen interviews, and the challenges relevant to data reduction (say, topical sorting) quickly become apparent. To render data manipulation manageable, I used a program designed for the computer-assisted analysis of text-based data, THE ETHNOGRAPH. ${ }^{1}$

THE ETHNOGRAPH automates what many fieldworkers know as "cut and paste." Suppose you have a pile of interview transcripts which you wish to sort into categories by subject or theme. In the good old days, this involved sitting down with stacks of photocopies and a pair of scissors, and manually cutting out pieces from all of the different interviews which pertained to a particular topic, after which these were "pasted" together to generate a single topically focused text; ergo, "cut and paste." Using ETHNOGRAPH, a series of interviews can be coded in such a way that all text pertaining to a particular topic or concept can be retrieved into a single file, and/or printed out.

To use the program, I transcribed each interview using my usual word processor, and reformatted the resulting text to half-page width 
(for reasons which will follow). Conversion into ASCII format then rendered the transcript "digestible" by ETHNOGRAPH. The next step in using ETHNOGRAPH is to "number and print" the file. This results in a printout of the transcript in which each line is numbered; the printout is confined to the left half of each printed-out page, the right half is blank. Now the printout is read, and sections of the transcript(s) labeled with codewords. This is called "code mapping."

Figure 1 shows an excerpt from a "numbered and printed" transcript. Lines 106 through 114 refer to war names; thus I penciled in a bracket enclosing these lines in the blank right-side half of the printout, and labeled it with the code word WAR. Secrecy - the reticence to mention one's name - occurs at lines 112-113; these I coded with SECRET. As I was also initially interested in generating a list of examples of Navajo names, I tagged lines 107-108 with EX. With this technique you can develop a list of code words and use them to label sections of text throughout as many interview transcripts as you are working with. Essentially, these codes identify topics; they constitute "retrieval and organizing devices that allow the analyst to spot quickly, pull out, then cluster all the segments relating to the particular question, hypothesis, concept, or theme" (Miles \& Huberman 1984, 56).

Figure 1. Part of a "numbered and printed" file, with "code mapping."

Females are given war names at

birth, an example being yilh

deezbaa $7^{2}$--She Going To War. One's

grandmother or mother usually give

it, though they tell it to the child

only when the child is old enough,

and they tell the child not to mention

it, only on special occasions. During

prayer, the medicine man asks for

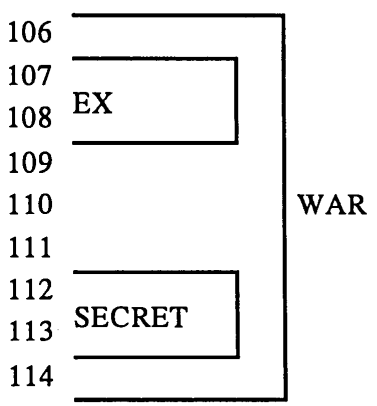

Often, one begins coding by noting, to the right of the numbered transcript(s), one's impressions or ideas, leads, topics, or other features discerned therein. Initial codes may be quite concrete and descriptive, though as a study progresses, they frequently become increasingly abstract and theoretical. Codes may be "empirically grounded" forthcoming from patterns or topics one sees in the data itself (the 


\section{Names 43.4 (December 1995)}

"grounded theory" approach), or they may be established prior to fieldwork on the basis of one's conceptual framework, hypotheses, or other variables (the "logical positivist" stance) ${ }^{3}$ I engaged a sort of middle ground, employing an initial "start list" of codes which I had assembled via literature review - codes such as PUBLIC (for Anglo or "public use" names), NICK (for nicknames), and WAR (for "war" or ceremonial names). I subsequently modified the coding scheme (via subdivisions and additions of codes) on the basis of what I "saw" in the data I had collected.

After completion of "code mapping," the numerous instances of coded text segments are easily entered into the computer by specifying the beginning and ending line numbers of the text segments associated with each code. Searches may now be conducted using single codes, co-occurrence of multiple codes, or multiple code searches which exclude segments which may be associated with a particular code word. A sample output is presented as figure 2, which shows how the numbered and coded file segment presented in figure 1 would appear after searching for segments encoded by WAR. The file name and line numbers insure that the context of the retrieved text segment is retained. Note also the date of the search, the page number (additional retrieved segments would occupy subsequent pages), and the manner in which the codes embedded within the retrieved segment (in this case EX and SECRET) are presented. ETHNOGRAPH thus serves those who wish to impose their own organizing scheme on a series of texts, after which it is easy to retrieve segments which thematically belong together.

My initial interviews concerned the refinement of a taxonomy of Navajo names. I began by employing my basic "start list" of descriptive codes. For example, all interview segments relevant to "baby" or "endearment" names were coded with BABY. ETHNOGRAPH allowed me to quickly assemble all of these excerpts into a single file, which allowed for easy construction of the abbreviated summary shown in figure 3 .

Endearment names such as kii ya7a7zh 'my little boy' were often used to address young children (these being some of the few names so employed given that kinship terms would generally be used for address in lieu of names themselves). These names are often called "baby" or "pet" names and are usually assigned by parents and/or grandparents. 
Figure 2. An example of a text segment retrieved by searching for segments coded by WAR.

SORTED OUTPUT FOR FILE NAV-ONE 5/30/1995

PAGE 1 SORT CODE: WAR

SC: WAR

\#--WAR

: Females are given war names at

106-\#

\$--EX

: birth, an example being yilh

$107 \quad \# \quad--\$$

: deezbaa7--She Going to War. One's

$108 \quad \# \quad-\$$

: grandmother or mother usually give

$109 \#$

: it, though they tell it to the child $110 \#$

: only when the child is old enough,

$111 \#$

\$--SECRET

: and they tell the child not to mention

: it, only on special occasions. During

$112 \#--\$$

$113 \#--\$$

Figure 3. A general taxonomy of Navajo names.

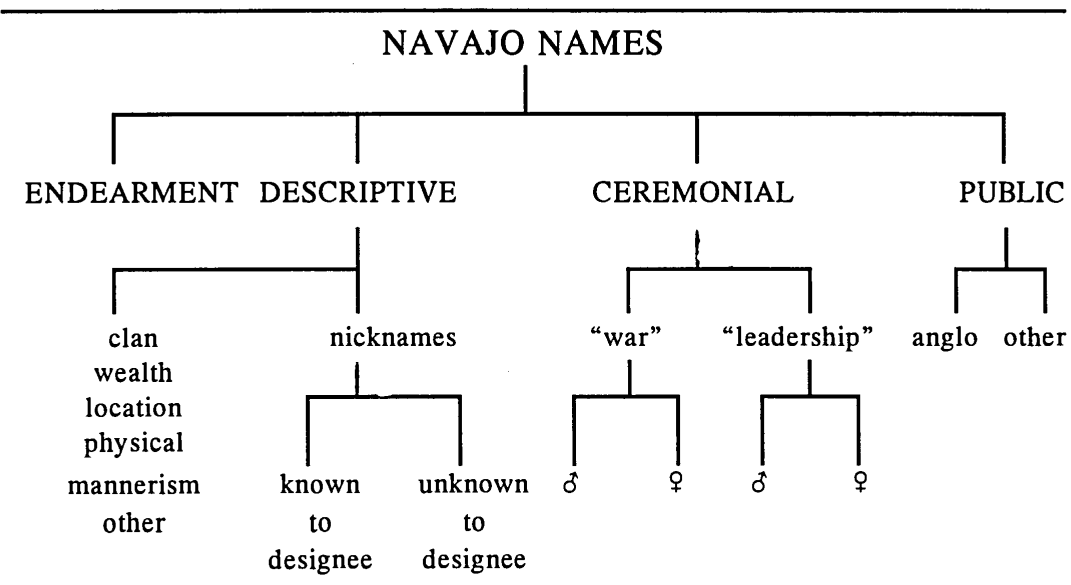

Although their use generally fades as the recipient grows older, endearment names could nonetheless be maintained, and used for instance by a grandparent to a grandchild (regardless of the latter's age). These terms could also be generated by using diminutive forms of the nouns such as 'asdz8a7a7 'woman', yielding 'asdz8a7a7 le 7i7 'little woman'. Furthermore, their pronunciation could employ different tones 


\section{Names 43.4 (December 1995)}

- perhaps roughly, tenderly, or in such a way as to make a child seem clumsy; the tone in a sense describing the personality of the individual to whose name it was appended. Note that the data suggest a number of additional themes for which I generated new codes, such as AGE (period of life when a name might be received), ORIGIN (origin of a name), or SOUND (sound or pronunciation of a name). These were added to my code list and employed to code subsequent interviews.

By a similar process, I conflated all of my transcripts tagged with codes such as DESC (descriptive names) and NICK (nicknames) into single files for each code, enabling efficient construction of a summary statement on "descriptive names." A large group of names might be considered descriptive in nature, being indicative of clan affiliation, wealth, residence location, physical or other qualities, such as sila7ago $n e 7 e 7 z$ 'tall policeman', which reflects occupation or physical characteristic. Often incorporating such common terms as biye' 'son of', bina7li7 'grandson', or bitsui 'daughter', these descriptive names usually originated decades ago as terms of reference for others; they were not intended for use in address. Many of these names have long since lost the immediacy of their connection to originating circumstances (a name meaning 'many mules' no longer connoting the ownership of animals), and translated into English, have solidified into family surnames which are now inherited as per Anglo conventions. Typical transcript excerpts follow, both here and throughout this article.

If there were just a few in an area that belonged...to the Kiiyaa'aanii clan, then he might be identified as the tall Kiiyaa'aanii Clansman, or the corpulent one, or the short one....

[He] had a crippled knee.... In Navajo they just called him na'nilhhodi7 'The One Who Limped'.... Everybody knew him by that name.

Nicknames constitute another genre of descriptive name, one which several of my consultants suggested bifurcated into two types: those which are known to the nicknamed person, and those which are not. These names may be used as a means of reference toward a particular individual in the community, or they may find informal use within a family. Not uncommonly, individuals would receive a series of nicknames throughout their lives. 
He [was] always making mistakes so...in the Navajo culture they'll say bihodiisht'e7e7h...[from which] his nickname Odie [was derived].

Can have a nickname for someone you don't like, sort of to chop him down to size. These arise within the family [your clan].... It can get out and people will call you that, but not directly. Such a name is a way to make public [within one's own kin] what you feel about another individual.

Again, the retrieved data suggested additional codes - such as ADD/REF (use for address and/or reference), LOC (references to location), etc. - which were incorporated into the code list.

In addressing ceremonial names - commonly called "war" or "secret" names - I had initially coded large chunks of data with basic codes like WAR and SECRET (reluctance to mention such a name). Data reduction required additional fine-tuning, and I subsequently added new codes suggested by what I saw in the data, codes such as CEREM (use of name in ceremony), ENEMY (Enemyway ceremony), HOLYP (Holy People), MED (medicine man), and so on. Retrieving these text segments into separate files for each code word allowed for the interrogation of discrete aspects of "war" naming, such as origin, ceremonial employment, etc. By summarizing each of these components, I efficiently assembled a general statement about such names, as shown in the following extracts.

Ceremonial or "war" names have both male and female versions (figure 3), reflecting a duality paradigmatic in Navajo culture:

Everything is divided into...male and female. East is male, west is female...the right side is our female side, the left side is our male side ...and all Navajo culture [is] based on [this duality]."

Retrieving data coded by MYTH (mythology) + WAR yielded transcripts relating to the mythological ancestry of such names, which derive from the two sons of Changing Woman: Monster Slayer and Born for Water. It is they, according to the Navajo creation story, who rid the world of the monsters which had arisen because of prior adulterous acts, after which the first Navajo were created.

Monster Slayer was a warrior... and Born for Water...did not do the actual killing. So there's a warrior side and there's a peaceful side.... Monster Slayer stands for the male, Born for Water represents the female. 


\section{Names 43.4 (December 1995)}

So...boys get their names from Monster Slayer, girls get their names [from] Born for Water... This is how ceremonial names are given.

To one not thoroughly accustomed to Navajo culture, it is difficult to grasp how such a name works. Realizing that thought in Navajo mythology is the source of creation, and speech the outer form of that thought, one gains some sense of the sacred creative nature of naming - of what one consultant meant when he stated that "to give such a name is to give something that people can fear from." Another, a Chanter (medicine man) stated that such a name (as well as the tone of one's voice) was recognized by "God" in somewhat the same way that the cry of a lamb becomes recognizable to its mother. Such a name, he felt, was like a password, the combination to a lock; it granted access, and should not be repeated needlessly because "disease people could learn to recognize it." There is a recognition among the Navajo that the nature of such names is but slowly revealed by time - the product of age, wisdom, and ceremonial participation.

A medicine man would not tell you everything unless he is very old. And then when he can no longer perform ceremonies, then he will tell his apprentice everything. A name is also the same way.

There is the possibility of another type of ceremonial name as well. One consultant felt that a "war" name like naat'a 7 yilh sida 7 'He sits making a speech' reflected qualities achieved later in life relevant to leadership. Perhaps a genre of ceremonial name, or an artifact of shifting perceptions relevant to "war" names, I tagged this with CUE to mark it for further research.

Retrieving interview data coded by ENG (English or Anglo influence) and PUBLIC (for publicly-used names), in combination with codes such as ORIGIN (how a name originated) and EX (a Navajo name example), I formulated the following statement relevant to "public names," those names used for reference and address in the modern world, generally one's English name.

Among the contemporary Navajo, the English name generally follows Anglo conventions. The first name reflects one's parents' preference, and the last name - in many cases the translation of a Navajo name - is now formalized and inherited as a family surname. Needless to state, the Navajo did not originally have English names, 
being instead identified by clan and kin affiliations. Increasing interface with Anglo culture (via schools, the census, traders, and other Anglo institutions) dictated that a reliable means of identification (from the Anglo perspective) be implemented. This of course meant that the Navajo had to have "proper" names according to Anglo conventions.

In retrieving the references I had coded by SCHOOL, it quickly became apparent that many of my consultants' Anglo surnames had originated via a grandparent who, as a child first attending school, had received an English name which was subsequently passed on as a family name. Furthermore, in retrieving transcripts coded by PUBLIC + ORIGIN (thus querying the origin of such names), it became apparent that Anglo names were usually generated by three means (sometimes in combination):

1. An attempt might be made to approximate the sound of the individual's Navajo name. For instance, a school teacher upon hearing the name hastiin biye' 'son of hastiin' would translate biye' into Begay, which would then become one's last name. (Note the imposition of the Anglo naming template, in that biye', coming as it did at the "end" of the name, was assumed to be a surname.) Similarly, bina7li7 'grandson' became Benally, and ya7zhi7 'small' became Yazzie. Such Anglicized names have now become very common family surnames.

2. A second avenue involved attempted translation of a Navajo name. For example, thi7zi7 lha7ni7 biye' 'son of Manygoats,' upon school enrollment, may simply receive Manygoats as a name.

3. Finally, there are many instances of assigning an English name which had no relation to one's Navajo name.

My husband's father while being examined for military service gave his Navajo name lh8i7i7 lhiba7hi7 'Gray Horse'. They couldn't handle that, so they gave him 'Wilson'.

And another source of names largely for adults was given by traders ... [who were sometimes] rather mean-humored. Because they gave people names that...oftentimes were ridiculous [names such as Bogus Check, Horse's Ass Begay, or Hastiin Goddamn, as recorded in Young \& Morgan, 1987].

The aforementioned examples demonstrate my utilization of ETHNOGRAPH. Simply put, I began with a basic list of descriptive codes which "chunked" my interview data into broad topics. Upon 


\section{Names 43.4 (December 1995)}

gaining more familiarity with the data, I recoded my interviews with an expanded series of codes which served to define additional topics, concepts, and themes. (My recoding employed a total of forty codes which proved surprisingly easy to assign after I got used to them.) This allowed for a rather fine-combed topical sorting of all of my interview transcripts, which in turn enabled my investigation of a wide range of subjects, such as the influence of school and religious institutions on naming practices, or problems of cultural interface occasioned by conflicting Anglo and Navajo naming templates.

As an example, I had noted many instances of name change - both short term (via particular individuals changing their names) and long term (trends in naming practices). These I tagged with the code NCHANGE. By searching my interviews with code combinations such as WAR + NCHANGE, or PUBLIC + NCHANGE, I generated richly suggestive files containing examples of trends and "name changes" for each of my name categories. For instance, searching my interviews for segments coded by PUBLIC and NCHANGE (in essence, querying the database as to changes or trends in Navajos' "public" or Anglo names) yielded transcripts such as this one:

The parents that...have very little education...[or] the first generation picking up English names...tend to pick up simple names, the generations of people that were born in the $60 \mathrm{~s}$, you know, have more [complex] names.

I became interested in the common-sensical intimation that the first generation to receive Anglo names got simpler ones, while later Anglo names evidenced greater complexity and I decided to follow this strand of evidence. Such a hypothesis seemed amenable to archival testing, and I thus recoursed to the genealogical and census files held by the Franciscan Mission at Fort Defiance, Arizona.

I thought that the qualitative interview evidence might be substantiated by quantitative data by comparing census figures from three different decades to evaluate name complexity and diversity. I began by examining a sample of two hundred Anglo first names each from the 1915 census (Chinle District), the 1940 census (Fort Defiance District, tabulated in 1939), and the late 1950s reservation school survey which included data from the 1940s and 1950s. For each sample population, I calculated the average number of syllables per name, as well as the average number of times the names were used (as a simple ratio, $\mathrm{X}$ per 
100 names listed). I performed similar calculations strictly for those names which appeared more than once, on the assumption that such names should constitute a more significant subset of the total sample. The results were then charted for the years 1915, 1939, 1945, and 1955. These are given in figure 4, which shows that the modal Anglo first name in 1915 had 1.5 syllables. This increased to 2.1 syllables by 1955 . Multiply-used names show a similar trajectory, increasing from 1.4 syllables in 1915 to 2.1 in 1955 . Concomitantly, the average number of uses per name was 2.2 per 100 in 1915 , declining to 1.3 per 100 in 1955; similarly for multiply-used names, which declined from 4.3 per 100 uses per name to 2.9 per 100 in 1955 . We see increasing syllabic complexity coupled with a reduction in frequency of use for any particular name - the latter occurring at a time when the incidence of Anglo naming among Navajos was greatly increasing (the percentage of individuals with Anglo names increased from 4.7\% in 1915 to $34.1 \%$ in 1940). This suggests that a much greater number of Anglo names were available from which to choose. Thus the increasing complexity and diversity of Anglo names suggested by my interviews appeared to be supported by the census figures.

Returning to the interview data and inspecting those segments which were retrieved under codes such as PUBLIC (public names) + NCHANGE (name change) or ENG (English Anglo influence), it appeared that the increasing frequency of Anglo names reflected increasing contact with, and acculturation to, Anglo culture - a (not surprising) qualitative suggestion which, again, might be subjected to archival scrutiny. Noting that the census figures listed names, as well as school attendance and house vs. hogan residence, I employed a basic statistical test of association (Chi-square) which may be used to evaluate the possibility of relationship between two variables.

An exhaustive survey of the 1915 Chinle Census District figures yielded 192 Anglo names for Navajos which could definitively be associated with residence type, and 1,040 which could be associated with school attendance. The results were compelling. Anglo first names correlated with house residence, and Navajo first names with hogan residence (significant at the .001 level). Not surprisingly, school attendance also correlated with possession of an Anglo name, while nonattendance correlated with a Navajo-only first name. The associations were again significant at the .001 level (meaning that the distributions evaluated would occur by chance only one time in a thousand). 
286 Names 43.4 (December 1995)

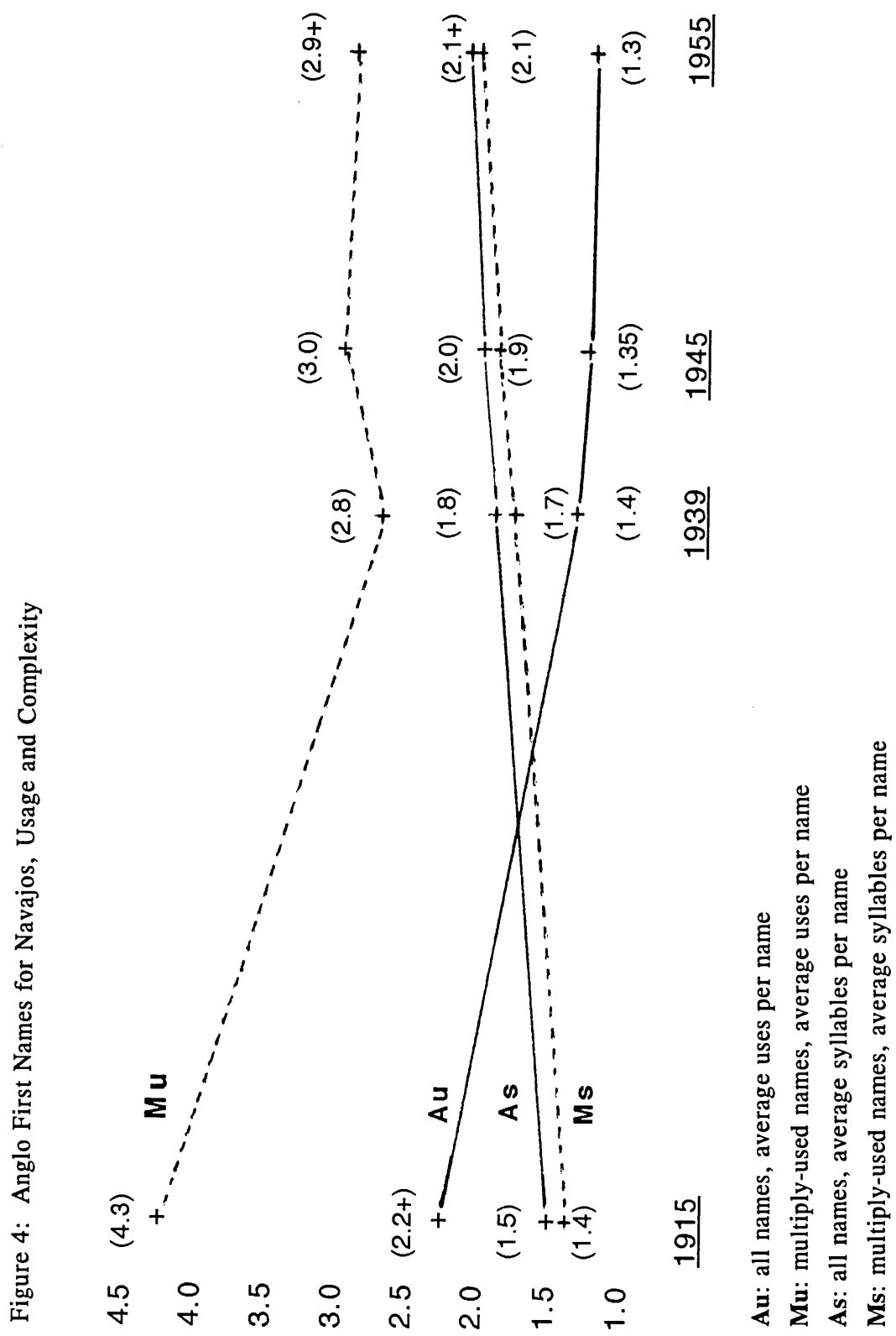


As Chi-square does not reveal the nature or strength of such relationships, additional recourse to interviewing would be called for. What I wish to illustrate is that even the most basic computerized manipulation of qualitative and quantitative data can prove richly rewarding in terms of hypothesis testing, allowing new lines of inquiry to be followed up while still in the field.

To further my investigation of naming as "process," I next looked more closely at "short term name change" - instances of name change at the personal level. Using ETHNOGRAPH, the researcher can easily return to the already-coded files in order to deploy new codes, and one of the codes I had added upon recoding my data was MOTIV, which tagged comments relevant to individuals' motives for changing their names.

Searching the interview files by MOTIV yielded the following motivations for name change:

1. To recognize a family change. ("He left his wife and family sit, took off with another...decided that's going to need another name"),

2. To clarify personal identity. ("They have names that are very common and they usually get mixed up"),

3. To confuse personal identity. ("When the law enforcement roster got too long"),

4. To take a more appropriate name. ("His father died two months before birth.... It was [not] appropriate for him to be named after someone who had died prematurely young"),

5. To express relationships. ("I...added her name to mine...so that I could think good about [her]"),

6. To satisfy a personal preference. ("I always liked the name"). To be sure, such comments apply in varying degrees to the different "types" of names. One's Anglo name for instance was (is) most amenable to change, while ceremonial names can be considered permanent.

By retrieving text tagged with various combinations of codes (such as NCHANGE+AGE, NCHANGE+SCHOOL, etc.), I was able to compile instances of name changes at various periods of life and for 


\section{Names 43.4 (December 1995)}

various reasons. A composite example was then easily constructed, which served as a tool for further interviews (figure 5).

Figure 5: An example of Personal Name Change

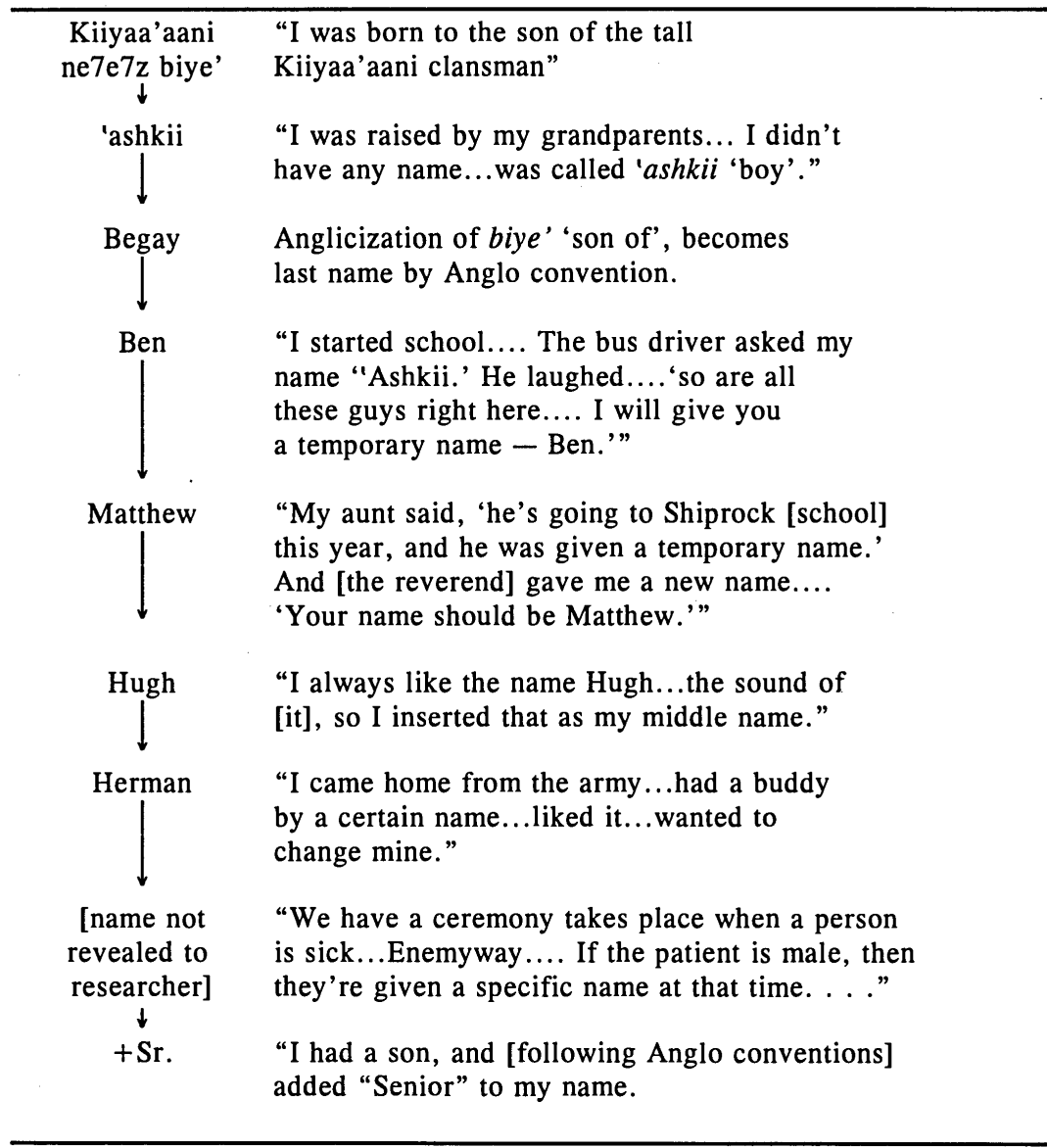

In this example, we see terms of endearment, temporary naming, religious influence, preference, ceremonial conference, and both traditional and Anglo conventions impinging upon the naming process. Through the processes described above, the individual who began life as Kiiyaa'aani ne $7 e 7 z$ biye' 'son of the tall clansman', became known instead as Herman Hugh Begay, Sr.

As noted previously, the first codes by which I categorized the data were concrete and descriptive. As fieldwork progressed, new codes 
became increasingly abstract, marking my discernment of emerging patterns and themes - codes such as REL (which tagged names' contributions toward the maintenance of one's relationship with kin, society, the Holy) or PARA (which carried intimations of paradigm, worldview). I offer the following as an example.

The Navajo have always been adept at integrating elements from other cultures into their own. Modern day religious practices - exhibiting major Anglo influence - exemplify this trait. Interview data provocatively suggested that such influences have impacted naming in a dynamic and deep-seated way, changing not so much the overt patterns or types of names, but the significance accorded them. In accessing those texts I had coded for WAR (ceremonial names), NCHANGE (trends), REL, and PARA, a tantalizing example of this conceptual shift was assembled.

Traditionally, ceremonial names were used within a paradigm which harbored no conception of good and evil per se as opposing forces "out there somewhere," rather, these qualities were immanent in all things - a balance obtaining. In disrupting that harmony, one might experience negative consequences, for which a ceremony might be required to effect rebalancing. The ceremonial name, as I understand it, served as a means of connection to those "forces" or "personifications" in the world - a means of identification "so that the Holy People would know you" - thus enabling reharmonization. But with the impact of "other influences [Christianity]...that's where the understanding has changed to a point where now that people talk about [the Anglo] God.... Our [traditional] God has no power." Thus with the ascendancy of Anglo religious conventions comes a different paradigm in which opposing diametric forces of "good" and "evil" contest against each other. A different relationship therefore obtains between the individual and the "Holy" - a connection formerly mediated via the vector of ceremonial names:

Christian people are the ones that have influenced [us]...[and] even traditional people somehow got this into their beliefs: there is evil and God. Two contradictory forces, that hunt for you. Our [traditional] God has no power [now].... You think there is evil and the names are not that much value, because these forces that you [pretend] are here... have more power over your name...so in a way that [the name] is not really necessary nowadays. 


\section{Names 43.4 (December 1995)}

This suggests a paradigm shift in the signification accorded ceremonial names. For while such names still appear to function as a "connection" between the individual and the Anglo God (in Christianized Navajo ceremony), they seem not to entail the web of relationships (to the Holy People, personified forces, earth, cosmology) that they once did. A valuable currency in a world of deeply inter-connected and balanced "forces," they seem, quite simply, to be overpowered in the Christian world of diametrically opposed, if not "omnipotent," powers. New conventions have broached a different way of perceiving the world, resulting in a changed, if not devalued, currency. Thus the qualitative evidence suggests a massive reconfiguration of the constellations of significance spun about the Navajo ceremonial name. Such data open up rich fields for further research.

I hope that I have illustrated several themes. First, that despite the inherent messiness and proliferation of paper attendant to interviewbased qualitative research - this brief exploratory study having yielded over 700 pages of notes - computerization, by mechanizing data manipulation as I have demonstrated here, can render the detail manageable and make revealing analysis possible. The data reduction from which this essay is excerpted was accomplished while still in the field, thus allowing for the feedback of provisional findings into the ongoing research process. And while ETHNOGRAPH is the most widely used software for the type of qualitative analysis I have illustrated, a number of other programs are also available. TEXTBASE ALPHA ${ }^{\circledR}$, TEXT ANALYSIS PACKAGE ${ }^{\circledR}$, QUALPRO $^{\circledR}$, and HYPERQUAL $^{\circledR}$ are all available from Qualis Research Associates (see note 1). These programs differ mainly in the combinations of options they offer, such as on-screen coding, direct entry of data without a word processor, ability to embed contextual information (speakers, notes on nonverbal interactions during interviews, etc.), ability to calculate word frequencies, use of different search strategies, and so on. (Tesch 1990 provides a detailed description of each program.) All of these allow for the manipulation of any kind of textual data in a way which databases themselves cannot. It is not necessary to "break down" one's text into "fields" prior to data entry, and the only data which are retrieved during a search is that which is relevant as per the segments defined via one's coding scheme - which can easily be developed or changed during the course of the analysis. And while I used ETHNOGRAPH primarily to 
organize and reduce interview transcripts, the program is equally amenable to the manipulation of textual data from diaries or books, questionnaire responses, or other written documentary or archival materials. Numerous applications may occur to the onomastician, ranging from the management of fieldnotes and the compilation of literature reviews, to the amalgamation into single files of, say, geographic name data which has been accumulated from a variety of sources.

Second, I have attempted to preserve some measure of how my own assumptions and "discoveries" in the field acted to shape the course of my initially unstructured study. In an age when knowledge-construction is acknowledged to be an artifact of one's interaction with the data, the subjective presence of researchers may render their reasoning and methodology more explicit. If it does so, asserts Morgan (1983), the resulting document - despite the appearance of subjectivity - may be considered more, and not less, scientific. This is an important consideration, for as Murray has argued $(1994,73)$, if onomasticians are to enhance their acceptance within the academic community, they must pay more attention to issues relevant to methodological rigor.

Finally, I wish to have seduced the reader with what are, to me, the rich though ill-defined rewards of interview-based onomastics - a mode of research greatly facilitated by ETHNOGRAPH. Sometimes one must point the finger at what cannot be directly stated:

He came to tell me about a name - biye' 'son of':

That's a real hard word to live by.... You have to respect yourself.... You have to kinda go according to whatever the teachings that your father has, whatever disciplines that he stresses...to a point where he would appreciate you. So that's what biye' means.... [He put out his cigarette and reached for his hat]. We journey through this life, on this little Mother Earth, this planet it's like a spaceship, its going through this universe. And the reason we're on this earth is...like a training ground. How are we going to conduct ourselves in the universe, when we go out there into the universe, if we don't learn how to conduct ourself on this earth? Somebody calls you son, that's a hard name to take care of.

And that is what he wanted to tell me about names - that steeped in the attitudes and values of a particular worldview, they carry a way of life with them. He gave me a big hug, turned and walked out the 


\section{Names 43.4 (December 1995)}

door. His words haunt me yet. Is this not the field onomastician's true reward? - to achieve what a searching of archives or crunching-of-numbers can rarely yield; that sense of having glimpsed, in studying one's fellow man, that which "concerns him most intimately...the hold which life has on him" (Malinowski 1961, 25).

\section{Notes}

1. I used ETHNOGRAPH 3.0 in my research; version 4.0 is now available. ETHNOGRAPH requires a 286 or later IBM compatible computer, uses $2 \mathrm{MB}$ of hard disk space, and needs at least 2MB RAM. It can search up to 200 data files (say, interview transcripts) at one time for occurrences of up to 100 codes. ETHNOGRAPH is available from Qualis Research Associates, 413-256-8835, for about $\$ 200.00$. Other text analysis programs are available from the same source.

2. Alas, the use of computers may present problems which handwriting does not. The transcription of Navajo terms requires marks and accents which most word processing programs cannot generate. Thus, I used standard computerized transcription conventions in which, as in the following examples and throughout this paper "7" indicates a tone, " 8 " a nasal, 1 is represented by " $\mathrm{h}$," and so on. Thus 'asdzáá léí 'little woman' is rendered 'asdz8a7a7 le7i7, and ttizi táni 'many goats' becomes thi 7zi7 lha7ni7; readability is surely compromised.

3. Readers unfamiliar with the methodologies pertinent to coding specifically, or qualitative research in general, may consult books on research methods such as Miles \& Huberman (1984), Strauss \& Corbin (1990), or one of many others. I have found a number of Sage publications to be particularly useful sources.

\section{References}

Bureau of Indian Affairs. 1961. The Navajo Yearbook: A Decade of Progress 1951-1961. Gallup, NM: Department of the Interior.

Malinowski, Bronislaw. 1961. Argonauts of the Western Pacific. New York: E. P. Dutton, [1922].

Miles, Matthew B. and A. Michael Huberman. 1984. Qualitative Data Analysis. Newbury Park, CA: Sage Publications.

Morgan, Gareth. 1983. Beyond Method. Newbury Park, CA: Sage Publications.

Murray, Thomas E. 1994. "On the Need to Legitimize Onomastics in Academia." Names 42: 67-78.

Reichard, Gladys A. 1928. Social Life of the Navajo Indians. New York: Columbia UP. . 1950. Navajo Religion: A Study of Symbolism. New York: Pantheon.

Strauss, Anselm and Juliet Corbin. 1990. Basics of Qualitative Research. Newbury Park, CA: Sage Publications.

Tesch, Renata. 1990. Qualitative Research: Analysis Types \& Software Tools. New York: The Falmer Press. 
Words to Live By 293

Young, R. W. and William Morgan. 1987. The Navajo Language. Albuquerque: $\mathrm{U}$ of New Mexico P. 